\title{
Independently outgrowing neurons with a geometric synapse formation model develop realistic network connectivity patterns with small-world properties
}

\author{
Andrew Carnell, Sander de Ridder, Jaap van Pelt, Arjen van Ooyen \\ From Twentieth Annual Computational Neuroscience Meeting: CNS*2011 \\ Stockholm, Sweden. 23-28 July 2011
}

In neural networks, connectivity plays an important role in cognition, while the geometry of neuronal arborisation is an important determinant of connectivity. However, precisely how synaptic connectivity depends on the detailed morphology of neurites remains poorly understood. The aim of this paper is to study the connectivity patterns of neural networks comprising neurons with realistic morphologies. These networks are created with our modelling tool NetMorph [1], a simulation framework that simulates neuronal morphogenesis from the perspective of the individual growth cone in a stochastic phenomenological manner. NetMorph has been shown to create realistic neuronal morphologies, [1].

The growth of networks of layer $2 / 3$ neurons was simulated using NetMorph. A realistic minimum neuron separation of 20 micron was used. The method used for synapse formation combines the proximity of axon and dendrite line-pieces [2] with a 'crossing criterion', [3].

A suite of various connectivity measures was used to analyse emergent network connectivity including analysis of synaptic distributions, small-world properties and of specific network shape parameters. Small-world topologies have been observed in numerous brain regions and allow for segregated as well as integrated processing. They are associated with both high local and high global efficiency and have low wiring cost and this makes them attractive models for the brain. It is shown that small-world connectivity is observed in all analysed NetMorph generated networks. The work in this paper

\footnotetext{
* Correspondence: andrew.carnell@cncr.vu.nl
Integrative Neurophysiology, Center for Neurogenomics and Cognitive

* Correspondence: andrew.carnell@cncr.vu.nl
Integrative Neurophysiology, Center for Neurogenomics and Cognitive Research (CNCR), VU University Amsterdam, De Boelelaan 1085, 1081 HV Amsterdam, The Netherlands
}

(c) 2011 Carnell et al; licensee BioMed Central Ltd. This is an open access article distributed under the terms of the Creative Commons

demonstrates that shape parameters, such as Euclidean distances from synaptic locations to postsynaptic somata, produce distributions that are highly similar to those distributions produced by experimentally observed networks. Our preliminary findings indicate that realistic neuronal morphologies, simple geometry-based synapse formation rules and independently developing neurons are capable of producing networks with realistic synaptic distributions, connectivity patterns and small-world properties.

\section{Acknowledgements}

This work was supported by EU MC-RTN NEURoVERS-it (019247), and BIO-ICT Project SECO (216593).

Published: 18 July 2011

\section{References}

Koene RA, Tijms B, van Hees P, Postma F, de Ridder A, Ramakers GJA, vanPelt J, van Ooyen A: NETMORPH: A Framework for the Stochastic Generation of Large Scale Neuronal Networks With Realistic Neuron Morphologies. Neuroinformatics 2009, 7(3):195-210.

2. Peters A, Kara DA, Harriman KM: The neuronal composition of area 17 of rat visual cortex. III. Numerical considerations. Journal of Computational Neurology 1983, 238(3):263-74.

3. van Pelt J, Carnell A, de Ridder S, Mansvelder HD, van Ooyen A: An algorithm for finding candidate synaptic sites in computer generated networks of neurons with realistic morphologies. Front. Comput. Neurosci 2010, 4:148.

doi:10.1186/1471-2202-12-S1-P356

Cite this article as: Carnell et al:: Independently outgrowing neurons with a geometric synapse formation model develop realistic network connectivity patterns with small-world properties. BMC Neuroscience 2011 12(Suppl 1):P356. 